\title{
Novel understanding on genetic mechanisms of enteric neuropathies leading to severe gut dysmotility
}

\author{
Francesca Bianco, ${ }^{1,2}$ Giulia Lattanzio, ${ }^{1}$ Luca Lorenzini, ${ }^{3}$ Chiara Diquigiovanni, ${ }^{2}$ Maurizio Mazzoni, ${ }^{1}$ \\ Paolo Clavenzani, ${ }^{1}$ Laura Calzà, ${ }^{3}$ Luciana Giardino, ${ }^{3}$ Catia Sternini, ${ }^{4}$ Elena Bonora, ${ }^{2 *}$ Roberto De Giorgio ${ }^{5 *}$ \\ ${ }^{1}$ Department of Veterinary Sciences, University of Bologna, Italy \\ ${ }^{2}$ Department of Medical and Surgical Sciences, University of Bologna, Italy \\ 3IRET Foundation, Ozzano Emilia, Italy \\ ${ }^{4}$ UCLA/DDRC, Division of Digestive Diseases, Departments Medicine and Neurobiology, David Geffen School of \\ Medicine, UCLA, Los Angeles, CA, USA \\ ${ }^{5}$ Department of Translational Medicine, University of Ferrara, Italy \\ *These authors share co-last authorship
}

The enteric nervous system (ENS) is the third division of the autonomic nervous system and the largest collection of neurons outside the central nervous system (CNS). The ENS has been referred to as "the brain-in-thegut" or "the second brain of the human body" because of its highly integrated neural circuits controlling a vast repertoire of gut functions, including absorption/secretion, splanchnic blood vessels, some immunological aspects, intestinal epithelial barrier, and gastrointestinal (GI) motility. The latter function is the result of the ENS fine-tuning over smooth musculature, along with the contribution of other key cells, such as enteric glia (astrocyte-like cells supporting and contributing to neuronal activity), interstitial cells of Cajal (the pacemaker cells of the GI tract involved in neuromuscular transmission), and enteroendocrine cells (releasing bioactive substances, which affect gut physiology). Any noxa insult perturbing the ENS complexity may determine a neuropathy with variable degree of neuro-muscular dysfunction. In this review we aim to cover the most recent update on genetic mechanisms leading to enteric neuropathies ranging from Hirschsprung's disease (characterized by lack of any enteric neurons in the gut wall) up to more generalized form of dysmotility such as chronic intestinal pseudo-obstruction (CIPO) with a significant reduction of enteric neurons. In this line, we will discuss the role of the RAD21 mutation, which we have demonstrated in a family whose affected members exhibited severe GI dysmotility. Other genes contributing to gut motility abnormalities will also be presented. In conclusion, the knowledge on the molecular mechanisms involved in enteric neuropathy may unveil strategies to better manage patients with neurogenic gut dysmotility and pave the way to targeted therapies.

Key words: Chronic intestinal pseudo-obstruction; enteric neuropathies; genes; Hirschsprung's disease; neuroprotection; 5-HT4 receptors.

Correspondence: Roberto De Giorgio, Department of Translational Medicine, Internal Medicine Unit, Arcispedale St. Anna, Via A. Moro 8, 44124 Ferrara, Italy. Tel. +39.0532.236631. E-mail: dgrrrt@unife.it

Contributions: All authors contributed equally to this manuscript 


\section{Introduction}

The digestive system contributes significantly to the maintenance of body homeostasis ${ }^{1}$ via a number of key physiological functions, including generating motor patterns to propel foods through the lumen of the gastrointestinal (GI) tract, mixing ingesta with different secretions, and absorbing nutrients. To serve this vast repertoire of coordinated activities, the GI tract requires finely tuned regulatory mechanisms, with the intrinsic innervation of the gut or enteric nervous system (ENS), being the most prominent one. ${ }^{2}$ Because of its ability to operate independently from central nervous system (CNS) and the "brain-like" organization, the ENS is also referred to as 'the brain-in-the-gut' or the 'second brain'. Notably, the ENS is composed by as many neurons as those present in the mammalian spinal cord (i.e., approximately $10^{8}$ ), making it the largest division of the autonomic nervous system (the other being the parasympathetic and sympathetic components). ${ }^{3}$

Research performed over the past four decades by enteric neuroscientists has shown that in the two main ganglionated plexuses of the ENS, namely the myenteric (Auerbach's) one (embedded in the longitudinal and circular layers of the GI tract) and submucosal (Meissner's) one (localized throughout the submucosa) there are functionally distinct classes of neurons. ${ }^{4}$ These include motor neurons, interneurons, vasomotor neurons and secretomotor neurons synaptically linked in reflex circuits controlling "canonical" (e.g., motility, absorption/secretion and splanchnic vasculature) and "non-canonical" functions (e.g., neuro-immune interactions and epithelial barrier integrity) via the release of a cocktail of neuromodulators and neurotransmitters and related receptors. ${ }^{5,6}$ The neurochemistry of the ENS prompted studies aimed at identifying novel pharmacological targets and therapeutic options, a typical example being 5-hydroxytryptamine (serotonin) and related receptors. ${ }^{7}$ Furthermore, likewise the CNS, the ENS possesses a prominent glia component exhibiting morphological and functional features to some extent similar to the astrocytes of the CNS. ${ }^{8-10}$

Enteric neuropathies are challenging conditions characterized by a severe impairment of gut physiology, including motility. Overall, the clinical manifestations and their severity increase as the enteric neuron number decreases. A recent study from our group suggests that a $50 \%$ loss of neuronal cell bodies in the myenteric and submucosal ganglia ( $v s$ controls) may be considered a "critical threshold" to the occurrence of clinical manifestations, such as recurrent intestinal sub-occlusive episodes related to impaired motility and severe symptoms (e.g., nausea, vomiting) ${ }^{11}$

Based on this background, the present review will focus on some aspects of genetically driven molecular abnormalities of enteric neuropathies ranging from Hirschsprung's disease, a condition characterized by lack of any enteric ganglia in the gut wall, up to more generalized dysmotility, such as CIPO characterized by an overall reduced number of enteric neurons. Deciphering the molecular mechanisms involved in enteric neuropathies may unveil strategies to optimize the treatment of patients with severe gut dysmotility and related symptoms.

\section{Hirschsprung's (HSCR) disease: a paradigm for complex diseases}

An extreme form of ENS neuropathy is aganglionosis, i.e., the absence of any enteric neurons and ganglia in the GI tract. This can occur as an acquired condition in about $20-30 \%$ of patients with Chagas disease (which is due to the parasite Trypanosoma cruzi infecting humans via Triatominae, a subclass of insects also known as "kissing bugs" common in the tropical area) or as a congenital, genetically-driven, disorder in HSCR, which affects approximately 1:5000 newborn. ${ }^{12,13}$ The classic clinical phenotype of HSCR is the lack of both myenteric and submucosal ganglia in the GI tract (usually the distal segments) as a result of an impaired enteric neuron migration and differentiation along the gut during prenatal life. The pathophysiological consequence of aganglionosis is a complete absence of peristalsis, the main motor behavior of the intestine leading to dilatation of the gut (a typical example being megacolon). Patients with HSCR require a rapid diagnosis and a surgical treatment aimed to remove the aganglionic segment.

HSCR is a disorder with genetic heterogeneity, but most cases present with mutations in the RET (rearranged during transfection), affecting either coding or non-coding parts of the gene. ${ }^{14}$ Other mutated genes, such as EDNRB and $S O X 10$, have an important impact on RET signaling thereby causing HSCR. In the last few years different genes and variants have been identified in association with this disease. ${ }^{15}$ Rapid technological advancements in molecular genetics, including genome-wide association studies (GWAS), whole exome sequencing (WES) and next generation sequencing (NGS) contributed to detect new pathological variants in patients with HSCR. ${ }^{15,16}$ In view of the considerable knowledge in this field, that described HSCR as a model of complex multigenic disorders, the new causative variants have been summarized in Table 1. The reader is referred to in-depth review on this topic that is beyond the scope of this work.

\section{Chronic intestinal pseudo-obstruction: under- standing the molecular features of gut dysmotility}

Chronic intestinal pseudo-obstruction (CIPO) is a very severe form of gut dysmotility, which manifests with recurrent sub-

Table 1. Major genes with causative variants, which have been found in Hirschsprung disease.

\begin{tabular}{llc} 
Gene & Gene name & Map position \\
RET & Ret proto-oncogene & $10 \mathrm{q} 11.21$ \\
NRTN & Neurturin & $19 \mathrm{p} 13$ \\
\hline GDNF & Glial Cell Derived Neurotrophic Factor & $5 \mathrm{p} 13$ \\
GFRal & Glial cell line-derived neurotrophic & $10 \mathrm{q} 26$ \\
& factor family receptor 1 & \\
\hline PSPN & Persephin & $19 \mathrm{p} 13$ \\
EDNRB & Endothelin Receptor Type B & $13 \mathrm{q} 22$ \\
\hline ECE-1 & Endothelin Converting Enzyme 1 & $1 \mathrm{p} 36$ \\
EDN3 & Endothelin 3 & $20 \mathrm{q} 13$ \\
\hline NTF3 & Neurotrophin 3 & $12 \mathrm{p} 13.31$ \\
NTRK3 & Neurotrophic Receptor Tyrosine Kinase 3 & $15 \mathrm{q} 25.3$ \\
\hline SOX10 & SRY-box containing gene 10 & $22 \mathrm{q} 13$ \\
L1CAM & L1 cell adhesion molecule & Xq28 \\
\hline NRG1 & Neuregulin 1 & $8 \mathrm{p} 12$ \\
PHOX2B & Paired-like homeobox 2b & $4 \mathrm{p} 13$ \\
\hline ZEB2 & Zinc Finger E-Box Binding Homeobox 2 & $2 \mathrm{q} 22.3$ \\
KIAA1279 & Kinesin family binding protein; KIFBP & $10 \mathrm{q} 22.1$ \\
\hline SEMA3A & Semaphorin 3A/3C/3D & $7 \mathrm{q} 21.11$ \\
SEMA3C/ & & \\
SEMA3D & & $20 \mathrm{q} 11.21$ \\
DNMT3B & DNA Methyltransferase 3 Beta & $4 \mathrm{p} 12$ \\
\hline PMX2b & Paired like homeobox 2b & \\
\hline & & \\
\hline
\end{tabular}


obstructive episodes, in the absence of any evidence of mechanical causes occluding the intestinal lumen. ${ }^{17} \mathrm{CIPO}$ can be either "secondary" to a wide array of recognized pathological conditions or "idiopathic" i.e., forms related to unknown etiology. ${ }^{18}$ So far, the management of CIPO patients remain largely unsatisfactory, thus leading to frustration for the patients, their families and physicians. Ideally, restoring GI motility may result in an improvement of the nutritional status and preserve the patient from a number of lifethreatening outcomes (septicemia being one of the most common complications). ${ }^{19,20}$ Likewise aganglionosis in HSCR, we will address idiopathic CIPO and, specifically, focus on genetic abnormalities. Combined clinical, histopathological and genetic studies are eagerly awaited to identify new perspectives in the understanding of CIPO. This is of utmost importance for classifying CIPO and establishing correlations between histopathological/clinical phenotypes and underlying genetic defects.

A general view of the causative genetic variants associated to CIPO has been highlighted in Figure 1 showing the different locations in the gut of the gene products involved in CIPO. The reader should be aware that although CIPO can originate from smooth muscle abnormalities ${ }^{21-24}$ and changes to interstitial cells of Cajal (ICC - the pacemaker cells of the GI tract), herein, we will cover the genetically-driven forms of neuropathy-related (or "neurogenic") CIPO. Thorough in vitro and in vivo studies of gene variants are required to understand their impact in severe enteric dysmotility of CIPO patients. In this line, the discovery of mutated genes represents the first step for novel targeted therapeutic strategies aimed at overcoming downstream molecular impairments underlying severe gut dysmotility. The next paragraphs will deal with RAD21, GOL1, POLG, TYMP and other new gene-related neuropathies.

\section{SGOL1 and RAD21}

Homozygous mutations in SGOL1 and RAD21, encoding for cohesin complex components, were identified in patients with CIPO. In 2014, Chetaille et al. ${ }^{25}$ described a new syndrome affecting gut and heart rhythm caused by a SGOL1 mutation. They

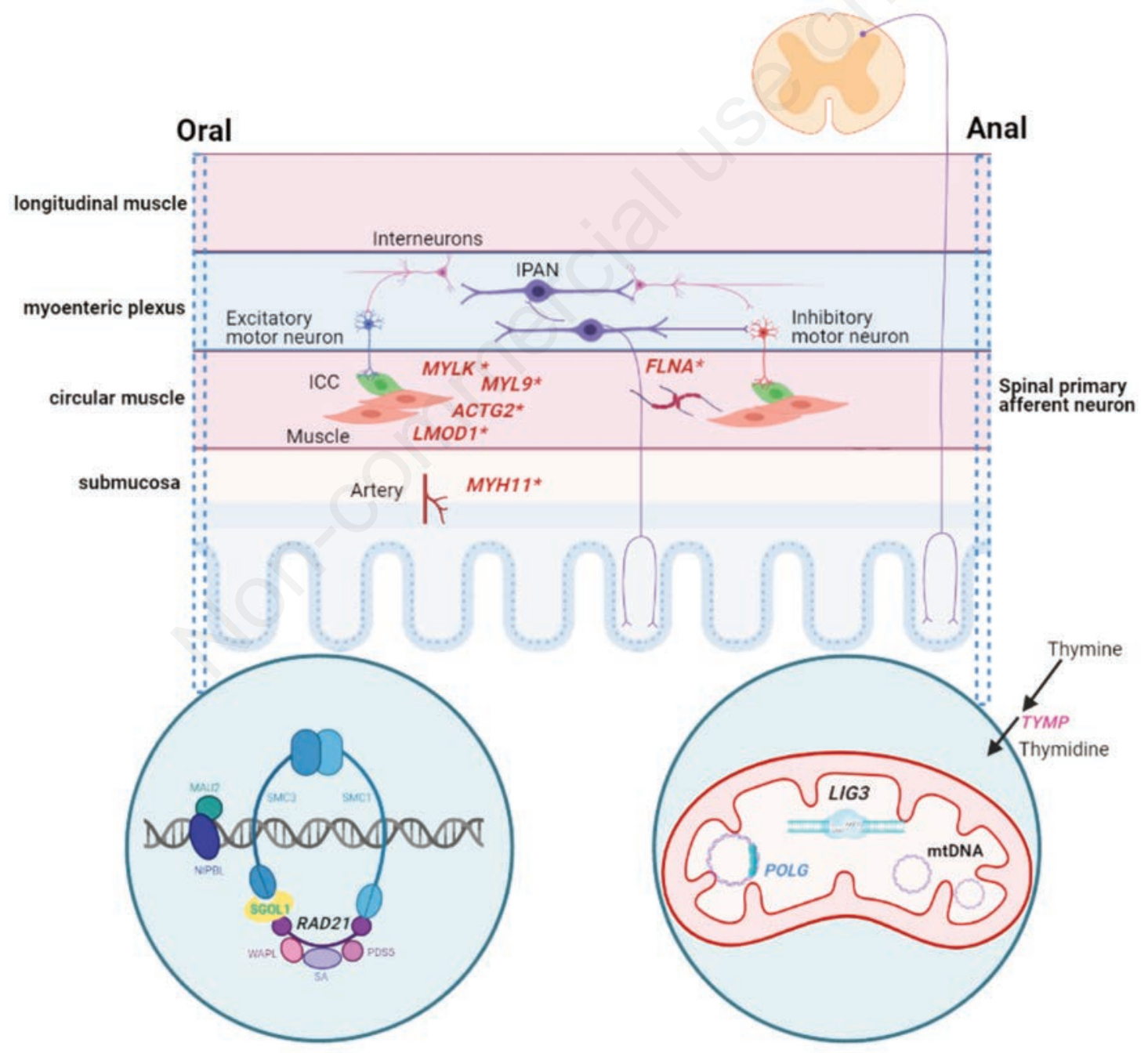

Figure 1. Possible topography of some genetic variants involved in gut neuro-muscular impairment and related dysmotility. MYLK, Myosin light chain kinase; MYL9, Myosin light chain 9; ACTG2, Enteric smooth muscle actin Y-2; LMOD1, leiomodin 1; MYH11, Myosin heavy chain 11; FLNA, filamin A; RAD21, Cohesin Complex Component; SGOL1, Shugoshin-like 1; TYMP, Thymidine phosphorylase gene; POLG, DNA polymerase gamma; LIG3, ligase III gene. IPAN, intrinsic primary afferent neuron; ICC, interstitial cells of Cajal; * genes associated to myopathic forms of CIPO. Created with BioRender. 
defined this condition as chronic atrial and intestinal dysrhythmia (CAID) syndrome, i.e., a novel generalized dysrhythmia, indicative of a new role for SGOL1 in mediating the integrity of human cardiac and gut rhythmicity, the latter being generated by ICC. Since SGOL1 is part of the cohesin complex, its dysfunction could entail consequences for long-range transcriptional regulation, possibly interfering with the expression of gene products associated with CIPO.

In the same period, our group identified a homozygous causative variant in a large consanguineous family segregating an autosomal recessive form of CIPO. In the affected family members, the major clinical feature was CIPO, in addition to megaduodenum, long-segment Barrett esophagus and cardiac abnormalities of variable severity (OMIM 611376; also referred to as 'Mungan syndrome', MGS). We performed WES analysis on the genomic DNA from two affected individuals and found the novel homozygous change c.1864G $>$ A in RAD21 (NM_006265.2) producing the damaging missense variant p.Ala622 $\mathrm{Thr}^{-}{ }^{-6}$ Likewise SGOL1, also RAD21 is part of the cohesin complex and is specifically involved in pairing and unpairing of sister chromatids during cell replication and division, as well as in the regulation of gene expression directly and independently of cell division. ${ }^{27}$ The RAD21 subunit of the cohesin complex plays important structural and functional roles as it provides the physical link between the SMC1/SMC3 heterodimer and the STAG subunit, thus affecting the association or disassociation of functional cohesin with chromatin.

In our in vivo study we could recapitulate in the zebrafish gut the CIPO phenotype, i.e., marked hypoganglionosis and severe impairment of motility, both features observed in the patients with the homozygous RAD21 mutation. After injecting a morpholino (mo) specific for the functional ablation of rad21, zebrafish embryos were allowed to develop to 5 days post fertilization (dpf), when the digestive system developed. Control and mo-treated embryos were fed fluorescent beads through microgavage, a technique that allows to assess the rate of intestinal motility as a function of time. After eight hours post bead injection, gut embryos were divided into four zones based on anatomical landmarks and the fluorescence detectable in each segment was scored. Compared to controls, rad21 morphants showed delayed food transit along the gut. Furthermore, a significant depletion of enteric neurons, detected with antibodies to the neuronal marker $\mathrm{HuC} / \mathrm{D}$, was quantitatively shown in the gut of $\operatorname{rad} 21$ morphants $v s$ controls. Taken together the marked impairment of gut motility along with the significant reduction of $\mathrm{HuC} / \mathrm{D}$-immunolabeled enteric neurons provided evidence of a neurogenic type of dysmotility reminiscent CIPO observed in patients with RAD21 mutations. ${ }^{26}$

The distribution of RAD21 in the mouse small intestine was another important step to better understand the RAD21-related functional implications in the ENS. In the mouse small intestine Rad21 immunoreactivity (IR) was detected in a subset of PGP9.5and $\mathrm{HuC} / \mathrm{D}$-immunolabeled myenteric neurons, specifically in $42.5 \%$ of $\mathrm{HuC} / \mathrm{D}-\mathrm{IR}$ neurons/field, and same percentage of $\mathrm{HuC} / \mathrm{D}-\mathrm{IR}$ neurons/field in young mice. A subset of choline acetyl transferase (ChAT, a marker of cholinergic excitatory neurons)positive neurons also expressed Rad21-IR. ${ }^{28}$ These data provided the basis to study how the RAD21 mutation affects the ENS by developing a genetically re-constructed $\operatorname{Rad} 21$ conditional knockin $(\operatorname{Rad} 21 \mathrm{KI})$ mouse carrying the Ala626Thr mutation found in affected family member. This model is expected to recapitulate the main clinical and pathological features observed in the affected RAD21 variant family members. The preliminary results showed an overall reduction of $\mathrm{HuC} / \mathrm{D}$ myenteric neurons/field in Rad21KI compared to wild type (WT) control mice $(\approx 20 \%$ in the ileum and $\approx 30 \%$ in the colon). We also showed that both $\mathrm{HuC} / \mathrm{D} / \mathrm{ChAT}$ - and $\mathrm{HuC} / \mathrm{D} /$ neuronal nitric oxide synthase, nNOS - a marker of inhibitory neurons) myenteric neurons/field were reduced in Rad21KI than in WT mice (Bianco et al., unpublished data). The reduction of neuronal populations represents a mechanism underlying the impairment of motility in CIPO patients with the RAD21 mutation. Further studies are in progress to determine the full spectrum of abnormalities in this mouse model.

\section{TYMP, POLG, RRM2B: mitochondrial disorders and gut neuro(myo)pathy}

The mitochondrial neurogastrointestinal encephalomyopathy (MNGIE) is an autosomal recessive disorder due to TYMP, POLG and $R R M 2 B$ mutations. ${ }^{29}$ In addition to a severe derangement of GI motility (i.e., gastroparesis and CIPO) resulting from an underlying enteric neuro-myopathy, ${ }^{11,30}$ the clinical picture is characterized by peripheral neuropathy, ophthalmoplegia and brain leucoencephalopathy detectable at magnetic resonance imaging. TYMP encodes for the thymidine phosphorylase (TP) enzyme, which regulates the mitochondrial nucleotide pool. TP is a cytoplasmic enzyme expressed in most human tissues including the central and peripheral nervous systems, the GI tract, leukocytes, liver and platelets. $^{31}$ TP catalyzes the first step of mitochondrial dThd and dUrd catabolism by converting them to the nucleotides thymine and uridine, respectively, and 2-deoxy ribose 1-phosphate..$^{32}$ Thus, TYMP mutations evoke TP dysfunction leading to accumulation of both dThd and dUrd and a subsequent reduction of cytidine triphosphate (dCTP) in the plasma and tissues of MNGIE patients. This imbalance interferes with mtDNA replication, causing molecular abnormalities (mtDNA depletion, multiple deletions, and point mutations) and tissue damage (including the neuromuscular component of the GI tract) associated with the disease. Mutations in the gene coding for the catalytic subunit of the mitochondrial DNA (mtDNA) polymerase ( $P O L G)$ are associated with a range of clinical syndromes characterized by secondary mtDNA defects, including mtDNA depletion and multiple deletions. The clinical manifestations are closely resembling those of MNGIE, although brain leukoencephalopathy is absent.

Finally, compound heterozygous mutations in the gene encoding for the ribonucleotide reductase regulatory TP53 inducible subunit M2B $(R R M 2 B)$ can cause a phenotype, characterized by ophthalmoplegia, ptosis, GI dysmotility, cachexia, peripheral neuropathy, clinically indistinguishable from TYMP mutated MNGIE.

\section{What is new in mitochondrial disorders: the $L I G 3$ mutation enteropathy}

We recently characterized another mutant gene in seven patients with CIPO and neurological manifestations (reminiscent of MNGIE) from three unrelated families. ${ }^{33}$ In addition to CIPO, the most prominent and consistent clinical signs were neurological abnormalities, including leukoencephalopathy, epilepsy, migraine, stroke-like episodes, and neurogenic bladder. DNA from these patients was examined via WES. Compound heterozygous variants were identified in the LIG3 gene in all patients. All variants were predicted to have a damaging effect on LIG3 protein. The LIG3 gene encodes the unique mitochondrial DNA (mtDNA) ligase that binds POLG and plays a pivotal role in mtDNA repair and replication. The study of the consequences of LIG3 mutations was performed in patient-derived primary skin fibroblasts and in transiently transfected cells expressing the different mutant $v S$ WT proteins. All assays showed a markedly reduced amount of LIG3 protein in the mutant cells. In concordance, we demonstrated the lack of lig- 
ase activity in the mitochondrial extracts derived from patients' cells compared to control fibroblasts. The LIG3 gene defects altered the mitochondrial network ${ }^{33}$ and affected mtDNA maintenance leading to mtDNA depletion without the accumulation of multiple deletions observed in other mitochondrial disorders (e.g., MNGIE) and induced a severe imbalance in cell metabolism (i.e., impaired ATP production and increased mitochondrial reactive oxygen species generation). The resultant mitochondrial dysfunction was key in the causative effects leading to the clinical phenotype observed in these patients. In the gut, both traditional histopathological analysis and neuronal $\mathrm{HuC} / \mathrm{D}$ immunoreactivity evaluation demonstrated a significant loss of the number of myenteric neuronal cell bodies in the gut (Figure 2). Furthermore, the lig3 disruption in the zebrafish model reproduced some key clinical features observed in the patients, i.e., brain leukoencephalopathy and impaired gut transit, with altered mitochondrial networks. Thus, biallelic heterozygous loss-of-function variants in the LIG3 gene resulted in a novel mitochondrial disease characterized by predominant gut dysmotility due to enteric neuropathy, and encephalopathy.

\section{Future perspective}

NGS has made available the simultaneous analysis of multiple genomic regions and reduced the time (and cost) of genetic tests. The discovery of new genes is of paramount importance for a better understanding of enteric neuropathies and related severe gut dysmotility. Also, this research is expected to develop novel therapeutic targets for affected patients who typically represent a challenge for their complex clinical picture. The lack of ENS models for studying the physio-pathological processes of enteric neuropathies detected in patients may account for the relatively slow progress in the diagnosis and minimally effective treatment options for these difficult cases.

In recent years, however, new technological progresses cast hope for disentangling the complexity of enteric neuropathies. In fact, using the direct differentiation of human induced pluripotent stem cells (PSCs), it has been possible to obtain physiological three-dimension organ cultures, known as organoid..$^{33} \mathrm{~A}$ recent study applied a tissue-engineering approach with embryonic and
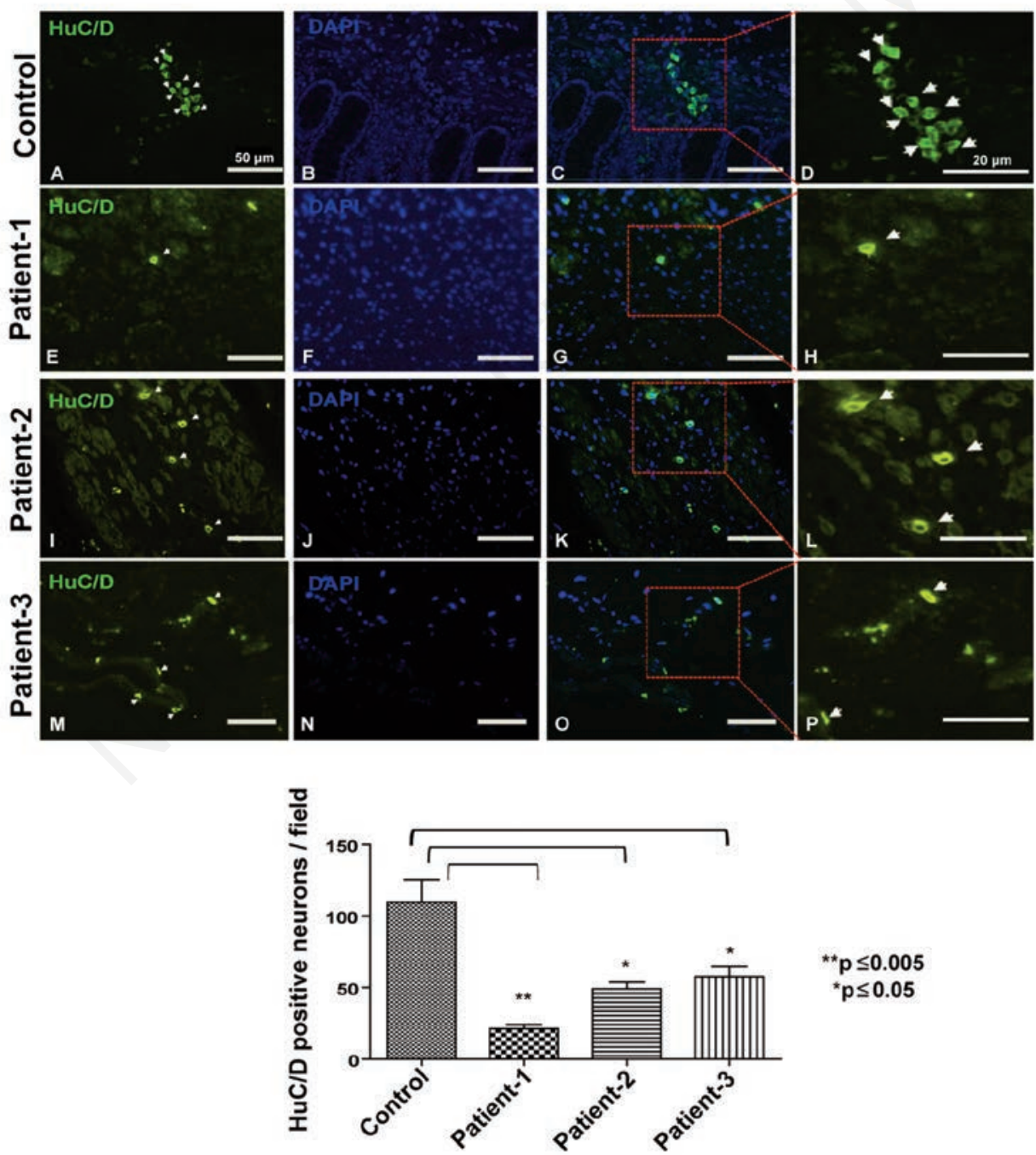

Figure 2. Mutated LIG3 mitochondrial-related entero-neuropathy. In the cryostat sections, few HuC/D immunolabeled (green) myenteric cell bodies (arrows) were observed in the gut (colon) biopsies of patients with LIG3 causative variants vs controls. Cell nuclei were stained with DAPI (blue). Quantitative analysis (graph below) confirmed that myenteric neurons were significantly reduced in patients carrying the causative variants in $L I G 3$, compared to controls ( $\mathrm{p} \leq 0.005$ control vs patient-1 and $\mathrm{p} \leq 0.05$ control vs patient-2 and 3 ; ANOVA). Scale bar: $50 \mu \mathrm{m}$. Photomicrographs in D, H, L and P illustrate higher magnification insets of the indicated region. Scale bar: $20 \mu \mathrm{m}$. 
PSC to generate a human intestinal tissue containing a functional ENS. The normal ENS development was recapitulated in vitro by combining human-PSC-derived neural crest cells and developing human intestinal organoids (HIOs), with migration to the mesenchyme, differentiation into neurons and glial cells, and generation of neuronal activity. ${ }^{34}$ The ENS-containing HIOs in vivo formed neuro-glial structures similar to myenteric and submucosal plexuses, exhibited functional ICCs and generated waves of propagating contractions. The same approach was applied to study PHOX $2 B^{-/}$related HSCR mouse model studied extensively by Workman et al. ${ }^{35}$ The authors developed a functional intestinal tissue that could be transplanted in vivo and highlighted the potential of stem cell-based therapies for future treatment of severe gut dysmotility. Another frontier of ENS research concerns the molecular profiling, regional distribution, and relative conservation across species of various enteric neuron subsets (mainly the myenteric plexus). Emerging research has provided novel insights on marker genes specific for the different enteric neuron classes (ENCs). Recently, Morarach et al. ${ }^{36}$ addressed this extremely innovative area and provided molecular evidence of twelve ENCs within the myenteric plexus of the mouse small intestine. The authors identified inter-communication features and histochemical markers for discrete classes of neurons, including motor, sensory, and interneurons together with genetic tools for class-specific targeting. ${ }^{36} \mathrm{~A}$ recent work from Ganz et al. ${ }^{37}$ showed that genes controlling epigenetic modifications are important to coordinate intestinal tract development, providing the first demonstration that these genes influence ENS development. In this line, another study in human and mouse healthy tissues used laser-capture microdissection coupled to single-nucleus RNA-sequencing, to map out enteric neuron subtypes in the duodenum, ileum, and colon, which were overall conserved between humans and mice based on orthologous gene expression. Nevertheless, some enteric neuron subtype-specific genes in mice were expressed in completely distinct morphologically defined neuron subtypes in humans, thus suggesting that these species-specific differences should be taken into account when translating findings from mouse to human $\mathrm{ENS}^{38}$. Taken together these exciting results offer a conceptual and molecular resource for dissecting ENS circuits and predicting key regulators for the directed differentiation of distinct enteric neuron classes. Clearly, these data will change our approach and understanding of enteric neuropathy-related severe gut dysmotility.

\section{Conclusions}

In this review, we presented evidence that a thorough genetic analysis is a crucial approach to highlight the molecular pathways involved in ENS morpho-functional changes, hence enteric neuropathy, and severe gut dysmotility. Since current evidence suggests that the ENS may be viewed as a "dynamic system", characterized by continuous turnover of neurons (i.e., those "dying" being replaced by resident precursors), clarifying the genetic abnormalities affecting neuronal subclasses of the ENS may be a step forward to develop effective targeted therapeutic options for patients. Likewise to CNS disorders, the technological advances in genomics, molecular phenotyping and regenerative medicine (using pluripotent stem cells as possible treatment options) will represent the beginning of a new era in ENS disorders and related functional GI impairment.

\section{Acknowledgements}

Figure 1 has been created using BioRender.com.

\section{References}

1. Holland AM, Bon-Frauches AC, Keszthelyi D, Melotte V, Boesmans W. The enteric nervous system in gastrointestinal disease etiology. Cell Mol Life Sci 2021;78:4713-33.

2. Nick J Spencer, Hongzhen Hu. Enteric nervous system: sensory transduction, neural circuits and gastrointestinal motility. Nat Rev Gastroenterol Hepatol 2020;17:338-51.

3. Langley JN. On the reaction of cells and of nerve-endings to certain poisons, chiefly as regards the reaction of striated mus. cle to nicotine and to curare. J Physiol 1905;33:374-413.

4. Furness J.B. The enteric nervous system and neurogastroenterology. Nat Rev Gastroenterol Hepatol 2012;9:286-94.

5. Sternini C, Wong H, Pham T, De Giorgio R, Miller LJ, Kuntz $\mathrm{SM}$, et al. Expression of cholecystokinin a receptors in neurons innervating the rat stomach and intestine. Gastroenterology 1999;117:1136-46.

6. De Giorgio R, Barbara G, Furness JB, Tonini M. Novel therapeutic targets for enteric nervous system disorders. Trends Pharmacol Sci 2007;28:473-81.

7. Berger M, Gray AJ, Roth BL. The expanded biology of serotonin. Annu Rev Med 2009;60:355-66.

8. Ochoa-Cortes F, Turco F, Linan-Rico A, Soghomonyan S, Whitaker E, Wehner S, et al. Enteric glial cells: A new frontier in neurogastroenterology and clinical target for inflammatory bowel diseases. Inflamm Bowel Dis 2016;22:433-49.

9. Gulbransen BD, Sharkey KA. Novel functional roles for enteric glia in the gastrointestinal tract. Nat Rev Gastroenterol Hepatol 2012;9:625-32.

10. Neunlist M, Rolli-Derkinderen M, Latorre R, Van Landeghem L, Coron E, Derkinderen P, et al. Enteric glial cells: recent developments and future directions. Gastroenterology 2014; 147:1230-7.

11. Boschetti E, Malagelada C, Accarino A, Malagelada JR, Cogliandro RF, Gori A, et al. Enteric neuron density correlates with clinical features of severe gut dysmotility. Am J Physiol Gastrointest Liver Physiol 2019;317:G793-G801.

12. Knowles CH, Lindberg G, Panza E, De Giorgio R. New perspectives in the diagnosis and management of enteric neuropathies. Nat Rev Gastroenterol Hepatol 2013;10:206-18.

13. Pawolski V, Schmidt HHM. Neuron-glia interaction in the developing and adult enteric nervous system. Cells 2021;10:47.

14. Brooks AS, Oostra BA, Hofstra RM. Studying the genetics of Hirschsprung's disease: unraveling an oligogenic disorder. Clin Genet 2005;67 6-14.

15. Luzón-Toro B, Villalba-Benito L, Torroglosa A, Fernández MR, Antiñolo G, Borrego S. What is new about the genetic background of Hirschsprung disease? Clin Genet 2020;97:114-24.

16. Zhang Z, Li Q, Diao M, Liu N, Cheng W, Xiao P, Zou J, et al. Sporadic Hirschsprung disease: mutational spectrum and novel candidate genes revealed by nextgeneration sequencing. Sci Rep. 2017;7:14796.

17. De Giorgio R, Cogliandro RF, Barbara G, Corinaldesi R, Stanghellini V. Chronic intestinal pseudo-obstruction: clinical features, diagnosis, and therapy. Gastroenterol Clin North Am 2011;40:787-807.

18. De Giorgio R, Sarnelli G, Corinaldesi R, Stanghellini V. Advances in our understanding of the pathology of chronic 
intestinal pseudo-obstruction. Gut 2004;53:1549-52.

19. Joly F, Amiot A, Messing B. Nutritional support in the severely compromised motility patient: when and how? Gastroenterol Clin North Am 2011;40:845-51.

20. Di Nardo G, Di Lorenzo C, Lauro A, Stanghellini V, Thapar N, Karunaratne TB, et al. Chronic intestinal pseudo-obstruction in children and adults: diagnosis and therapeutic options. Neurogastroenterol Motil 2017;29:e12945.

21. Halim D, Wilson MP, Oliver D, Brosens E, Verheij JBGM, Han Y, et al. Loss of LMOD1 impairs smooth muscle cytocontractility and causes megacystis microcolon intestinal hypoperistalsis syndrome in humans and mice. Proc Natl Acad Sci USA 2017;114:E2739-47.

22. Moreno CA, Metze K, Lomazi EA, Bertola DR, Almeida Barbosa RH, Cosentino V, et al. Visceral myopathy: Clinical and molecular survey of a cohort of seven new patients and state of the art of overlapping phenotypes. Am J Med Genet A 2016;170:2965-74.

23. Batzir NA, Bhagwat PK, Larson A, Akdemir ZC, Bagłaj M, Bofferding L, et al. Recurrent arginine substitutions in the ACTG2 gene are the primary driver of disease burden and severity in visceral myopathy. Hum Mutat 2020;41:641-54.

24. Matera I, Bordo D, Di Duca M, Lerone M, Santamaria G, Pongiglione $\mathrm{M}$, et al. Novel ACTG2 variants disclose allelic heterogeneity and bi-allelic inheritance in pediatric chronic intestinal pseudo-obstruction. Clin Genet 2021;99:430-6.

25. Chetaille P, Preuss C, Burkhard S, Côté JM, Houde C, Castilloux $\mathrm{J}$, et al. Mutations in SGOL1 cause a novel cohesinopathy affecting heart and gut rhythm. Nat Genet 2014;46:1245-9.

26. Bonora E, Bianco F, Cordeddu L, Bamshad M, Francescatto L, Dowless $\mathrm{D}$, et al. Mutations in RAD21 disrupt regulation of APOB in patients with chronic intestinal pseudo-obstruction. Gastroenterology 2015;148:771-82.

27. Horsfield JA, Print CG, Mönnich M. Diverse developmental disorders from the one ring: Distinct molecular pathways underlie the cohesinopathies. Front Genet 2012;3:171.

28. Bianco F, Eisenman ST, Colmenares Aguilar MG, Bonora E, Clavenzani P, Linden DR, et al. Expression of RAD21 immunoreactivity in myenteric neurons of the human and mouse small intestine. Neurogastroenterol Motil 2018;30:e13429.
29. Filosto M, Cotti Piccinelli S, Caria F, Gallo Cassarino S, Baldelli E, Galvagni A, et al. Mitochondrial neurogastrointestinal encephalomyopathy (MNGIE-MTDPS1). J Clin Med 2018;7:389.

30. Giordano C, Sebastiani M, De Giorgio R, Travaglini C, Tancredi A, Valentino ML et al. Gastrointestinal dysmotility in mitochondrial neurogastrointestinal encephalomyopathy is caused by mitochondrial DNA depletion. Am J Pathol 2008;173:1120-8.

31. Boschetti E, D'Alessandro R, Bianco F, Carelli V, Cenacchi G, Pinna AD et al. Liver as a Source for thymidine phosphorylase replacement in mitochondrial neurogastrointestinal encephalomyopathy. Plos One 2014;9:e96692.

32. Martí R, López LC, Hirano M. Assessment of thymidine phosphorylase function: measurement of plasma thymidine (and deoxyuridine) and thymidine phosphorylase activity. Methods Mol Biol 2012;837:121-33.

33. Bonora E, Chakrabarty S, Kellaris G, Tsutsumi M, Bianco F, Bergamini $\mathrm{C}$, et al. Biallelic variants in LIG3 cause a novel mitochondrial neurogastrointestinal encephalomyopathy. Brain 2021;144:1451-66.

34. Lehmann R, Lee CM, Shugart EC, Benedetti M, Charo RA, Gartner Z, et al. Human organoids: a new dimension in cell biology. Mol Biol Cell 2019;30:1129-37.

35. Workman MJ, Mahe MM, Trisno S, Poling PH, Watson CL, Sundaram N, et al. Engineered human pluripotent-stem-cellderived intestinal tissues with a functional enteric nervous system. Nat Med 2017;23:49-59.

36. Morarach K, Mikhailova A, Knoflach V, Memic F, Kumar R, Li W, et al. Diversification of molecularly defined myenteric neuron classes revealed by single cell RNA-sequencing. Nat Neurosci 2021;24:34-46.

37. Ganz J, Melancon E, Wilson C, Amores A, Batzel P, Strader M, et al. Epigenetic factors Dnmt1 and Uhrfl coordinate intestinal development. Dev Biol 2019;455:473-84.

38. May-Zhang AA, Tycksen E, Southard-Smith NA, Deal KK, Benthal JT, Buehler DP, et al. Combinatorial transcriptional profiling of mouse and human enteric neurons identifies shared and disparate subtypes in situ. Gastroenterology 2021;160:755-70.

Received for publication: 11 June 2021. Accepted for publication: 3 November 2021.

This work is licensed under a Creative Commons Attribution-NonCommercial 4.0 International License (CC BY-NC 4.0).

(C) Copyright: the Author(s), 2021

Licensee PAGEPress, Italy

European Journal of Histochemistry 2021; 65(s1):3289

doi:10.4081/ejh.2021.3289 